\title{
Raucherbein vom Passivrauchen
}

\author{
Chefredakteur Dr. med. Dirk Einecke
}

COPD

\section{Wie sicher sind inhalative Anticholinergika?}

In der letzten Woche wurden widersprüchliche Daten zur Sicherheit einer COPD-Therapie mit inhalativen Anticholingergika veröffentlicht: Eine Metaanalyse mit knapp 15000 Patienten kam zu dem Schluss, dass Ipratropiumbromid und Tiotropiumbromid das Risiko für kardiovaskuläre Ereignisse (kardiovaskulären Tod, Herzinfarkt oder Schlaganfall) erhöhen. Dem widerspricht eine interne Untersuchung des Herstellers von Tiotropiumbromid, bei der über $19000 \mathrm{~Pa}$ tienten berücksichtigt wurden und die sogar einen protektiven Effekt auf das

\section{HOCHDRUCKTHERAPIE}

\section{Für das IOWiG bleiben Diuretika erste Wahl}

Das Institut für Qualität und Wirtschaftlichkeit im Gesundheitswesen (IOWiG) hat am 18. September seinen zweiten Vorbericht zur Primärtherapie der essentiellen Hypertonie veröffentlicht. Die Hauptbotschaft lautet unverändert: „Diuretika können als Therapie der ersten Wahl angesehen werden. "Begründet wird die Vorrangstellung der Diuretika damit, dass die Substanzklasse in den ausgewerteten 16 Studien im Hinblick auf die Vermeidung von Folgekomplikationen keiner anderen Wirkstoffgruppe unterlegen war. In einzelnen Aspekten gab es sogar Hinweise auf eine Überlegenheit von Diuretika gegenüber ACEHemmern (Schlaganfallprävention) und Kalziumantagonisten (Vermeidung einer Herzinsuffizienz).

Die diabetogene Wirkung der Diuretika wird in dem Bericht zwar bestätigt, nach Einschätzung des IOWiG lässt sich daraus aber „kein patientenrelevanter Schaden ableiten“. Die Empfehlung des IOWiG steht im Widerspruch zu den Empfehlungen der Deutschen Hochdruckliga, die für eine individualisierte Hochdrucktherapie plädieren, bei der Nebenwirkungen und Verträglichkeit der verschiedenen Substanzklassen stärker berücksichtigt werden sollten.
Herz-Kreislauf-System gefunden hat. Entwarnung geben auch die vorab veröffentlichten Sicherheitsdaten der UPLIFTStudie. In dieser Studie wurden die Langzeiteffekte von Tiotropiumbromid bei fast 6000 Patienten prospektiv untersucht. Hier war weder die Sterblichkeit insgesamt (Risk Ratio o,89) noch die kardiovaskuläre Mortalität (RR 0,73) gegenüber einer Placebotherapie erhöht. Ebenso wenig wurde beim Risiko für Herzinfarkt oder Schlaganfall ein Anstieg beobachtet (RR 0,73 bzw. 095).

JAMA 2008;300:1439-50

\section{EKZEM-PRÄVENTION}

\section{Warum Babys Fisch essen sollten}

Eine einzige Fischmahlzeit vor Erreichen des neunten Lebensmonats reduziert das Risiko, an Ekzemen zu erkranken, um $25 \%$. Dies fanden Epidemiologen der Universität Göteborg heraus, die Langzeitdaten von ca. 17000 Säuglingen sammelten. Für die protektive Wirkung der einen Fischmahlzeit ist es völlig unbedeutend, welche Art von Fisch (arm oder reich an Omega-3-Fettsäuren) gegessen wird. Arch Dis Child 2008; DOI: 10.1136/adc.2008.140418.

\section{EIGENSTÄNDIGER RISIKOFAKTOR}

\section{Auch Passivrauchen erhöht das PAVK-Risiko}

Frauen, die niemals geraucht haben, aber Rauch ausgesetzt sind, haben ein um etwa 50\% erhöhtes Risiko, eine periphere arterielle Verschlusskrankheit (PAVK) zu entwickeln. In einer Studie bei über 60jährigen Chinesinnen zeigte sich unabhängig von anderen Risikofaktoren ein signifikanter, dosisabhängiger Zusammenhang nicht nur zwischen dem Ausmaß der Passivrauch-Exposition und der Entwicklung einer koronaren Herzkrankheit und eines ischämischen Schlaganfalls, sondern auch einer PAVK.

Circulation 2008: DOI: 10.1161/ Circulationaha.108.784801

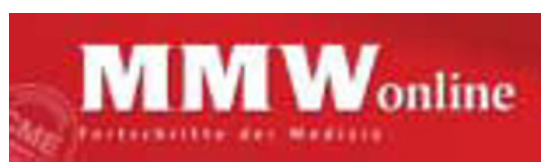

\section{GESETZLICHE FORTBILDUNGSPFLICHT}

\section{Der CME-Countdown läuft!}

Am 30. Juni 2009 schnappt die „Fortbildungsfalle“ zu: Können Sie als niedergelassener Arzt bis zu diesem Stichtag gegenüber Ihrer KV nicht nachweisen, dass Sie in den vergangenen fünf Jahren 250 Fortbildungspunkte gesammelt haben, ist die KV gesetzlich verpflichtet, Ihre Honorare aus der Vergütung vertragsärztlicher Tätigkeit zu kürzen. Im ersten Jahr nach dem Stichtag um 10\%, im zweiten um $25 \%$, und ab dem dritten Jahr um $100 \%$. Lassen Sie es gar nicht soweit kommen, und sammeln Sie Ihre Fortbildungspunkte mit der MMW: In einem Jahr erscheinen in der MMW über 30 CME-Fortbildungen, bei jeweils voller Punktzahl kommen so schon mehr als 90 CMEPunkte zusammen.

\section{Allergien in Deutschland}

Von je 1000 Bundesbürgern sind betroffen von:

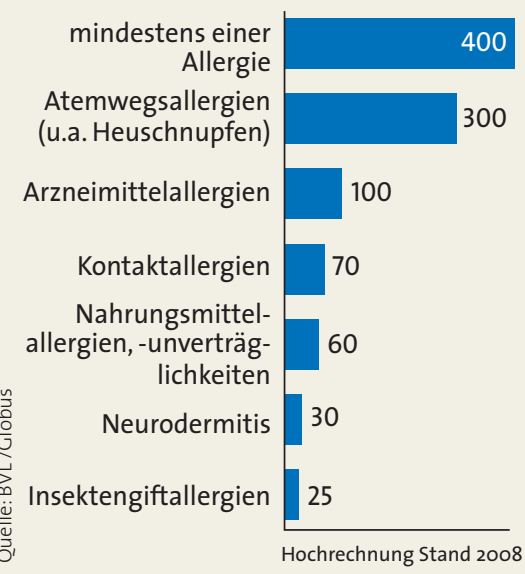

Allergien sind weiter auf dem Vormarsch. In Deutschland sind rund 40\% der Bevölkerung betroffen. In drei von vier Fällen handelt es sich um Atemwegsallergien. 